\title{
WAVE PROPAGATION IN THE LINEAR THEORY OF VISCOELASTICITY*
}

\author{
BY \\ GEORGE M. C. FISHER AND MORTON E. GURTIN \\ Brown University
}

1. Introduction. Recently Herrera and Gurtin [1] have shown that in the linear thoery of viscoelasticity the speed of propagation $U$ of an acceleration wave is a solution of the eigenvalue problem

$$
\left(G_{i j k l}(0) n_{j} n_{l}-\rho U^{2} \delta_{i k}\right) \beta_{k}=0 .
$$

Here $G_{i, k l}(0)$ is the initial value of the relaxation function $G_{i j k l}(t) ; \rho$ is the mass density; $\mathbf{n}$ is a unit vector in the direction of propagation of the wave.

In this paper we show that the eigenvalue problem (1.1) governs not only the speed of propagation of acceleration waves but also the propagation speeds of shocks and all higher order waves.

Finally we remark that the theory presented here is not empty. Indeed, within the context of linear viscoelasticity theory, Chu [2] has constructed a solution which has a first order discontinuity (shock). In addition, solutions which contain discontinuities of arbitrary order $N$ are easily exhibited.

Notation. Henceforth, $\mathbb{R}$ denotes an open region of Euclidean three dimensional space; $\mathbf{x}=\left(x_{1}, x_{2}, x_{3}\right)$ is a generic point of $R ; \beta$ is a regular subregion of $R$ (called a part of $R$ ); $\partial \rho$ is the boundary of $\rho ; \boldsymbol{n}$ is the outward unit normal to $\partial \rho$. The scalar $t$ denotes the time. Given a function $g$ of position and time we write $g^{(m)}$ for its $m$ th time derivative, i.e., $g^{(m)}(\mathbf{x}, t)=\partial^{m} g(\mathbf{x}, t) / \partial t^{m}$.

Letters in boldface denote vectors. We shall, for the most part, use indicial notation and Cartesian tensors. Thus subscripts have the range $(1,2,3)$; summation over repeated indices is understood; subscripts preceded by a comma indicate differentiation with respect to the corresponding Cartesian coordinate; $\delta_{i}$ designates Kronecker's delta. We shall sometimes find it convenient to use the vector cross product $\mathbf{x} \times \mathbf{y}$ and inner product $\mathbf{x} \cdot \mathbf{y}$.

2. The equations of linear viscoelasticity. The fundamental system of field equations for the linear theory of viscoelasticity consists of:

(i) the constitutive relation

$$
\sigma_{i j}(\mathbf{x}, t)=\int_{-\infty}^{t} G_{i j k l}(\mathbf{x}, t-s) d \gamma_{k l}(\mathbf{x}, s),
$$

where

$$
G_{i j k l}=G_{i j l k}=G_{i i k l} ;
$$

(ii) the strain displacement relation

$$
2 \gamma_{i j}(\mathbf{x}, t)=u_{i, j}(\mathbf{x}, t)+u_{i, i}(\mathbf{x}, t) ;
$$

*Received August 21, 1964; revised manuscript received October 22, 1964. 
(iii) the law of balance of linear momentum

$$
\frac{d}{d t} \int_{\mathcal{P}} \rho(\mathbf{x}) u_{i}^{(1)}(\mathbf{x}, t) d \mathbf{x}=\int_{\mathcal{P}} \rho(\mathbf{x}) f_{i}(\mathbf{x}, t) d \mathbf{x}+\int_{\partial \boldsymbol{P}} \sigma_{i i}(\mathbf{x}, t) \eta_{i}(\mathbf{x}) d \mathbf{x} .
$$

Here $\rho$ is the mass density, while $u_{i}, \gamma_{i j}, \sigma_{i i}, G_{i j k l}$, and $f_{i}$ are respectively the Cartesian components of the displacement vector $\mathfrak{u}$, the infinitesimal strain tensor $\gamma$, the symmetric* stress tensor $\boldsymbol{\delta}$, the relaxation function $\mathbf{G}$, and the body force density vector $\mathbf{f}$. The first two equations are assumed to hold at every $(x, t) \varepsilon R \times(-\infty, \infty)$; the third holds for every time $t$ and every part $\rho$ of $R$. We write the law of balance of linear momentum in integral form so as to include the possibility of discontinuous velocity and stress fields.

Finally we adjoin the initial condition

$$
u(\mathbf{x}, t)=0 \text { whenever }(\mathbf{x}, t) \varepsilon \cap \times(-\infty, 0) .
$$

3. Viscoelastic waves of order $\mathbf{N}$. Henceforth $S_{t}(0 \leq t<\infty)$ denotes a one-parameter family of surfaces with $S_{t} \subset R ; \Sigma_{S}$ is the hypersurface

$$
\Sigma_{S}=\left\{(\mathbf{x}, t) \mid \mathbf{x} \varepsilon S_{t}, 0 \leq t<\infty\right\} ;
$$

$\omega_{\mathbf{x}}$, for each $\mathbf{x}$, is the point set

$$
\omega_{\mathbf{x}}=\left\{t \mid \mathbf{x} \varepsilon S_{t}\right\}
$$

$N \geq 1$ is a fixed integer.

By a viscoelastic wave of order $N$ we mean a solution $\mathbf{u}, \boldsymbol{\gamma}, \boldsymbol{\delta}$ of (2.1), (2.2), (2.3), and (2.4) such that:

(A.1) The displacement $\mathbf{u}$ is $N-1$ times continuously differentiable on $R \times(-\infty, \infty)$; however its $N$ th order derivatives have jump discontinuities across $\Sigma_{S}$ but are continuous everywhere else on $R \times(-\infty, \infty)$. Moreover, each of these $N$ th order derivatives is bounded on every compact subset of $R \times(-\infty, \infty)$.

(A.2) The relaxation function $\mathbf{G}$ is $N$ times continuously differentiable on $a \times[0, \infty)$, while the body force $\mathrm{f}$ and the density $\rho$ are continuous and, if $N \geq 3, N-2$ times continuously differentiable on their respective domains $R \times(-\infty, \infty)$ and $R$.

(A.3) The hypersurface $\Sigma_{S}$ is smooth and orientable.

(A.4) For each $\mathbf{x}$, the set $\omega_{\mathbf{x}}$ has measure zero.

Condition (A.1) is the requirement that $\Sigma_{S}$ be a singular surface of order $N$ in the sense of Duhem and Hadamard.** Given a fixed point $(\mathbf{x}, t) \varepsilon \Sigma_{s}$, condition (A.3) implies the existence of a normal $v$ to $\Sigma_{S}$ at $(\mathbf{x}, t)$. Such a vector is

$$
\boldsymbol{v} \equiv(\mathrm{n},-U) \equiv\left(n_{1}, n_{2}, n_{3},-U\right), U \geq 0,
$$

where $\mathrm{n}=\left(n_{1}, n_{2}, n_{3}\right)$ is a unit normal to $S_{t}$ at the point $\mathbf{x}$. The vector $\mathbf{n}$ is called the direction of propagation; the number $U$ is called the speed of propagation. For $U \neq 0$

${ }^{*}$ The symmetry of the stress tensor insures that the angular momentum is balanced.

${ }^{* *}$ See Truesdell and Toupin [3], §187. 
we call any non-zero vector, which is parallel to the jump* $\left[\mathbf{u}^{(N)}(\mathbf{x}, t)\right]$, a wave axis. Roughly speaking, condition (A.4) asserts that the surface $S_{\mathfrak{t}}$, as it progresses, does not pass through a given point $x$ too many times.

Finally we use the following terminology: If $N=1$ we call the wave a shock wave**; if $N=2$ we call the wave an acceleration wave.

4. Shock waves. Our first theorem shows that the speed of propagation of a shock wave is governed by the same eigenvalue problem as that which arises in the study of acceleration waves.

Theorem 1. At a fixed point $(\mathbf{x}, t)$ the speed of propagation $U$ of a viscoelastic shock wave, whose direction of propagation is $\mathbf{n}$, satisfies the eigenvalue problem

$$
\left(G_{i j k l}(\mathbf{x}, 0) n_{j} n_{l}-\rho(\mathbf{x}) U^{2} \delta_{i k}\right) \beta_{k}=0 .
$$

Moreover, the eigenvector $\beta$ is an axis of the wave.

Proof. The integral statement (2.3) of the law of balance of momentum yields the well known jump condition

$$
-\rho U\left[u_{i}^{(1)}\right]=\left[\sigma_{i j}\right] n_{i} .
$$

If we integrate the constitutive relation (2.1) by parts and use the strain-displacement relation (2.2) together with the initial condition (2.4), we find that

$$
\sigma_{i j}(\mathbf{x}, t)=G_{i j k l}(\mathbf{x}, 0) u_{k, l}(\mathbf{x}, t)+\int_{0}^{t} G_{i j k l}^{(1)}(\mathbf{x}, t-s) u_{k, l}(\mathbf{x}, s) d s .
$$

Upon substituting (4.3) into (4.2) we arrive at

$-\rho(\mathbf{x}) U\left[u_{i}^{(1)}(\mathbf{x}, t)\right]=\left[G_{i j k l}(\mathbf{x}, 0) u_{k, l}(\mathbf{x}, t)+\int_{0}^{t} G_{i j k l}^{(1)}(\mathbf{x}, t-s) u_{k, l}(\mathbf{x}, s) d s\right] n_{i}$.

From Maxwell's Theorem ${ }^{\dagger}$ we know that the jump in the four dimensional gradient ${ }^{\ddagger}$ of the displacement $\mathfrak{u}$ is parallel to the hypersurface normal $\boldsymbol{v}$. Hence there exists a vector $\beta$ such that

$$
\left(\left[\frac{\partial u_{i}}{\partial x_{1}}\right],\left[\frac{\partial u_{i}}{\partial x_{2}}\right],\left[\frac{\partial u_{i}}{\partial x_{3}}\right],\left[\frac{\partial u_{i}}{\partial t}\right]\right)=\beta_{i}\left(n_{1}, n_{2}, n_{3},-U\right),
$$

or equivalently,

$$
\begin{aligned}
& {\left[\frac{\partial u_{i}}{\partial x_{i}}\right]=\beta_{i} n_{i},} \\
& {\left[\frac{\partial u_{i}}{\partial t}\right]=-\beta_{i} U .}
\end{aligned}
$$

${ }^{*}$ We use the usual notation for the jump $[g]$ of a function $g$ across $\Sigma_{s}$. That is $[g(\mathbf{x}, t)]=\lim _{\left(\mathbf{x}^{+}, t^{+}\right) \rightarrow(\mathbf{x}, t)} g\left(\mathbf{x}^{+}, t^{+}\right)-\lim _{\left(\mathbf{x}^{-}, t^{-}\right) \rightarrow(\mathbf{x}, t)} g\left(\mathbf{x}^{-}, t^{-}\right)$,

where $\left(x^{+}, t^{+}\right)$and $\left(x^{-}, t^{-}\right)$lie on opposite sides of $\Sigma_{S}$.

${ }^{* *}$ Actually, for $N=1$, it is customary to call the wave a shock wave if $\left[\mathfrak{u}^{(1)} \cdot \mathbf{n}\right] \neq 0$, a vortex sheet if $\left[\mathbf{u}^{(1)} \cdot \mathbf{n}\right]=0$. We do not make this distinction.

'See [3], §175.

${ }^{\ddagger} \mathrm{By}$ the four dimensional gradient we mean the operator $\left(\partial / \partial x_{1}, \partial / \partial x_{2}, \partial / \partial x_{3}, \partial / \partial t\right)$. 
Thus (4.6) and (4.7), when substituted into (4.4), yield

$$
\rho(\mathbf{x}) U^{2} \beta_{i}=G_{i j k l}(\mathbf{x}, 0) n_{i} n_{l} \beta_{k}+\left[\int_{0}^{t} G_{i j k l}^{(1)}(\mathbf{x}, t-s) u_{k, l}(\mathbf{x}, s) d s\right] n_{i} .
$$

Thus the proof reduces to showing that the jump in the integral in (4.8) vanishes. That such is the case is an immediate consequence of (2.1), (A.1), (A.2), (A.4), and the following lemma.

Lemma. Let $g$ and $k$ be functions on $R \times(-\infty, \infty)$ with the following properties:

(a) $g$ is continuous;

(b) $k$ is continuous everywhere except for a possible jump discontinuity across $\Sigma_{s}$;

(c) $k$ is bounded on every compact subset of $R \times(-\infty, \infty)$.

Moreover assume (A.4) holds. Then the function A defined by

$$
A(\mathbf{x}, t)=\int_{0}^{t} g(\mathbf{x}, t-s) k(\mathbf{x}, s) d s
$$

is continuous on $R \times(-\infty, \infty)$.

Proof. If we define

$$
B(\mathbf{x}, t, s)=g(\mathbf{x}, t-s) k(\mathbf{x}, s),
$$

then

$A\left(\mathbf{x}^{*}, t^{*}\right)-A(\mathbf{x}, t)=\int_{0}^{t} B\left(\mathbf{x}^{*}, t^{*}, s\right) d s+\int_{t}^{t *} B\left(\mathbf{x}^{*}, t^{*}, s\right) d s-\int_{0}^{t} B(\mathbf{x}, t, s) d s$.

We now choose $(\mathbf{x}, t) \varepsilon R \times(-\infty, \infty)$ arbitrarily, pick $T>0$ such that $(-T<t<T)$, and let $\Omega$ denote a closed sphere centered at $\mathrm{x}$ and contained in $R$. Further, we choose $\left(\mathrm{x}^{*}, t^{*}\right) \varepsilon \Omega \times(-T, T)$. By hypothesis, $B\left(\mathrm{x}^{*}, t^{*}, s\right)$ is bounded for

$$
\left(\mathbf{x}^{*}, t^{*}, s\right) \varepsilon \Omega \times[-T, T] \times[0, t] .
$$

Hence the second integral in (4.10) tends to zero as $\left(\mathbf{x}^{*}, t^{*}\right) \rightarrow(\mathrm{x}, t)$. Next, by (4.9) and our assumptions regarding $g, k$, and $\omega_{\mathbf{x}}$, we find that

$$
B\left(\mathbf{x}^{*}, t^{*}, s\right) \rightarrow B(\mathbf{x}, t, s) \quad \text { as } \quad\left(\mathbf{x}^{*}, t^{*}\right) \rightarrow(\mathbf{x}, t)
$$

for almost every $s \varepsilon[0, t]$. This fact and the boundedness of $B$, when used in conjunction with Lebesgue's Dominated Convergence Theorem, imply that

$$
\int_{0}^{t} B\left(\mathbf{x}^{*}, t^{*}, s\right) d s \rightarrow \int_{0}^{t} B(\mathbf{x}, t, s) d s
$$

and hence $A\left(\mathbf{x}^{*}, t^{*}\right) \rightarrow A(\mathbf{x}, t)$. This completes the proof.

5. Acceleration and higher order waves. Our main result is contained in the next theorem.

TheOREM 2. The speed of propagation $U$ of a viscoelastic wave is independent of the order $N$ of the wave and hence satisfies the eigenvalue problem (4.1), where again $\beta$ is an axis of the wave. 
Proof. By Theorem 1 it suffices to assume $N \geq 2$. For such a choice of $N$, (A.1) implies the $N-1$ compatibility relations

$$
\left[\frac{\partial}{\partial x_{s}}\left(u_{i, j k}^{(m)} \ldots_{l}\right)\right]=\left[\frac{\partial}{\partial t}\left(u_{i, j k \cdots l_{s}}^{(m-1)}\right)\right],
$$

where $j k \cdots l$ represent $q$ indices, $j k \cdots l s$ represent $q+1$ indices, and $q+m=N-1$.

Using Maxwell's Theorem for the jump in the four-dimensional gradient, we find that for each integer $m(1 \leq m \leq N-1)$ there exists an $m$ th order tensor ${ }^{(m)}[$ whose components satisfy

$$
\left[\frac{\partial}{\partial x_{s}} u_{i, j k}^{(m)} \ldots l\right]={ }^{(N-m)} \beta_{i j k} \ldots l n .
$$

and

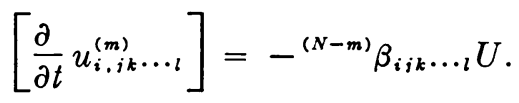

Thus (5.2), (5.3), and (5.4) imply

$$
-{ }^{(m+1)} \beta_{i j k} \ldots l_{\ell} U={ }^{(m)} \beta_{i j k} \ldots l n, ~,
$$

and hence

$$
{ }^{(m+1)} \beta_{i j k \cdots l s}(-U)^{m}=\beta_{i} n_{i} n_{k} \cdots n_{l} n_{s}\left(\beta_{i} \equiv{ }^{(1)} \beta_{i}\right),
$$

which, because of (5.3), yields

$$
(-U)^{N-m-1}\left[u_{i, j k}^{(m)} \cdots_{l}\right]=\beta_{i} n_{i} n_{k} \cdots n_{l} n_{s},
$$

where $j k \cdots l s$ indicate $N-m$ indices.

Next, since $N \geq 2$, (2.1), (2.2), and (2.3) imply the displacement equations of motion

$$
\begin{aligned}
\left\{G_{i j k l}(\mathbf{x}, 0) u_{k, l}(\mathbf{x}, t)\right\}_{, i}+\left\{\int_{0}^{t} G_{i j k l}^{(1)}(\mathbf{x}, t-s) u_{k, l}(\mathbf{x}, s) d s\right\}_{. i} & \\
& +\rho(\mathbf{x}) f_{i}(\mathbf{x}, t)=\rho(\mathbf{x}) u_{i}^{(2)}(\mathbf{x}, t) .
\end{aligned}
$$

We now differentiate (5.8) $N-2$ times with respect to position, take the jump in the resulting equation, and use (A.1), (A.2), and the lemma to verify that

$$
G_{i j k l}(\mathbf{x}, 0)\left[u_{k, l i p q} \ldots,(\mathbf{x}, t)\right]=\rho(\mathbf{x})\left[u_{i, p q}^{(2)} \cdots_{r}(\mathbf{x}, t)\right] .
$$

Consequently, (5.7) and (5.9) imply

$$
G_{i j k l}(\mathbf{x}, 0) \beta_{k} n_{l} n_{j} n_{p} n_{a} \cdots n_{r}=U^{2} \rho(\mathbf{x}) \beta_{i} n_{p} n_{a} \cdots n_{r} .
$$

Thus, if we multiply (5.10) by $n_{p} n_{q} \cdots n_{r}$ and use the fact that $n_{i} n_{i}=1$, we recover the eigenvalue problem (4.1) which completes the proof.

6. Longitudinal and transverse waves. We call a viscoelastic wave longitudinal if its direction is parallel to its axis, i.e., if

$$
n \times \underline{\beta}=0,
$$

transverse if its direction is perpendicular to its axis, i.e., if

$$
\mathbf{n} \cdot \underline{\beta}=0 .
$$


Theorem 3. Given a viscoelastic wave of order $N$, then the following three statements are equivalent:

(i) the wave is longitudinal;

(ii) $\left[\nabla \times \mathbf{u}^{(N-1)}\right]=0$;

(iii) $\boldsymbol{\beta}=\mathrm{n}\left[\nabla \cdot \mathrm{u}^{(N-1)}\right]$.

On the other hand, transverse waves are characterized by the next theorem.

Theorem 4. Given a viscoelastic wave of order $N$, then the following three statements are equivalent:

(i) the wave is transverse;

(ii) $\left[\nabla \cdot \mathbf{u}^{(N-1)}\right]=0$;

(iii) $\boldsymbol{\beta}=\left[\nabla \times \mathbf{u}^{(N-1)}\right] \times \mathbf{n}$.

Proof of Theorems 3 and 4. By (5.3)

$$
\mathbf{n} \cdot \hat{\beta}=\left[\nabla \cdot \mathbf{u}^{(N-1)}\right], \quad \mathbf{n} \times \boldsymbol{\beta}=\left[\nabla \times \mathbf{u}^{(N-1)}\right] ;
$$

and hence, the identity

$$
\boldsymbol{\beta}=\mathbf{n}(\mathbf{n} \cdot \underline{\beta})-\mathbf{n} \times(\mathbf{n} \times \beta)
$$

implies

$$
\boldsymbol{\beta}=\mathbf{n}\left[\nabla \cdot \mathbf{u}^{(N-1)}\right]-\mathbf{n} \times\left[\nabla \times \mathbf{u}^{(N-1)}\right] .
$$

Theorems 3 and 4 now follow as immediate consequences of (6.5). Equations (6.3) and (6.5) are sometimes referred to as Weingarten's First Theorem.*

7. Consequences of initial isotropy. In this section we suppress the variable $\mathbf{x}$ and write $G_{i j k l}(t) \equiv G_{i j k l}(\mathbf{x}, t)$ and $\rho \equiv \rho(\mathbf{x})$.

We call the relaxation function $G_{i j k l}(t)$ initially isotropic if $G_{i j k l}(0)$ is an isotropic tensor, i.e.,

$$
G_{i j k l}(0)=\frac{1}{3}\left(G_{2}-G_{1}\right) \delta_{i j} \delta_{k l}+\frac{1}{2} G_{1}\left(\delta_{i k} \delta_{i l}+\delta_{i l} \delta_{j k}\right) .
$$

The constants $G_{1}$ and $G_{2}$ are respectively the instantaneous shear modulus and the instantaneous modulus of pure compression.

The solutions to the eigenvalue problem (4.1), (7.1) are well known from linear isotropic elasticity theory. Thus we have the following theorem.

THEOREM 5. If the relaxation function is initially isotropic then there are two, and only two, possible speeds of propagation $\left(U_{L}\right.$ and $\left.T_{T}\right)$ of a viscoelastic wave. These speeds are given by:

(i) $U_{L}^{2}=\left(G_{2}+2 G_{1}\right) /(3 \rho)$ in which case the wave is longitudinal;

(ii) $U_{T}^{2}=G_{1} /(2 \rho)$ in which case the wave is transverse.

Conversely: if the wave is longitudinal, then $U=U_{L}$; if the wave is transverse, then $U=U_{T}$.

Acknowledgement. This paper is based on an investigation supported by the Office of Naval Research under Contracts Nonr 562(25) and Nonr 562(10). 


\section{REFERENCES}

[1] I. Herrera and M. E. Gurtin, A correspondence principle for viscoelastic wave propagation, Q. Appl. Math., 22 (1965) 360-364

[2] B. T. Chu, Stress waves in isotropic viscoelastic materials 1, Brown University, Division of Engineering, Contract ARPA, 1962

[3] C. Truesdell and R. A. Toupin, The classical field theories, Encyclopedia of Physics, Vol. III/1, Springer, Berlin, 1960 